\title{
Sometimes the Best Thing to do is Nothing: A Chiropractic Case Study
}

\author{
John Hart ${ }^{1,2 *}$ \\ ${ }^{1}$ Hart Chiropractic, Greenville, USA \\ ${ }^{2}$ Adjunct Faculty, Purdue University Global, USA
}

*Corresponding author: John Hart, Hart Chiropractic, Greenville, USA.

\begin{abstract}
The main purpose of care at Hart Chiropractic is to improve the function of the patient's autonomic nervous system (ANS). In some cases, the ANS is already functioning well even though the patient might have unpleasant symptoms. In such cases, the patient would not receive an intervention such as a spinal adjustment in this ANS-focused practice since the main objective is already met. This report highlights such a case where intervention was not necessary.
\end{abstract}

Keywords: Autonomic nervous system; Self-limited condition; Chiropractic

\section{Introduction}

Patient visits to clinicians typically include therapeutic intervention. When a clinician considers prescribing a treatment, he or she may also consider whether the patient's complaint might resolve on its own, as a self-limited condition that goes away without treatment. Such was the case with a recent new patient of mine whose complaint resolved without my service (of spinal adjustment).

In my practice, the purpose of care is to improve autonomic nervous system (ANS) function in the patient. (The terms neurological, autonomic, and ANS are used interchangeably in this paper.) This may seem like an unusual focus, but it is nonetheless an important one because the ANS plays a vital role in promoting a long and healthy life [1]. The ANS governs functions such as heart rate, digestion, respirator rate, and pupillary dilation / and constriction.

In this practice model, need for care is based on whether ANS function is diminished rather than on the presence of symptoms. These two considerations, autonomic function and presence of symptoms can be related in that improved ANS function can help patients heal themselves. However, there are times when the two do not coincide on a given visit. For example, the patient may have symptoms but ANS function is good. In such a case I have found that it is best to not to intervene [2].

The purpose of this case report is to provide an example of a patient not needing an intervention (of chiropractic adjustment) thereby sparing her time and money.

\section{Case Presentation}

Written consent for this case report was obtained from the patient. She is a young adult who sought care from me for her neck and shoulder pain that she had been experiencing for about 1-week prior. The study was declared exempt by the Institutional Review Board at Purdue University Global.

\section{Examination}

Autonomic function was assessed with resting heart rate (RHR) Carney RM, et al. [3], heart rate variability (HRV) [4-5] and bilateral 
infrared thermographic analysis for mastoid fossa differentials (MFD) [6].

Here is a snippet from the scientific literature supporting the use of RHR as an autonomic assessment: "Dysregulation of the autonomic nervous system is indicated by elevated resting heart rate" [7].

And for HRV: "Heart rate variability is used for non-invasive assessment of autonomic nervous system activity" [4].

And MFD: "Infrared thermography may represent an objective quantifiable indicator of autonomic disturbances" [8].

The heart rate measures were recorded simultaneously with the App, Heart Rate Variability Logger [9]. The App uses a sensor, the Kyoto ear clip which is based on photoplethysmography (PPG) technology and sends a Bluetooth signal to the App. The set-up has good agreement with standard ECG technology [10-11].

The HRV metric used was the root mean square of successive differences (rMSSD), a time-domain measure. The terms HRV and rMSSD are used interchangeably in this paper. For HRV a larger number is considered better than a smaller number, and for RHR a smaller number is generally better than a larger number.

The MFD was measured with a non-contact thermometer known as Thermofocus [12]. For MFD interpretation, a combination of symmetry and pattern interpretation was used, where change in symmetry (e.g., the warmer side changes from the left side to the right side) is considered neurologically healthier compared to persistent increased heat on the same side [6].

Alignment of the atlas (C1) vertebra (another area of focus in my practice) was assessed using manual palpation. The atlas transverse process on each side is compared to the ipsilateral tip of the mastoid process to determine if there is any lateral misalignment of atlas.

\section{Visit 1 of 2}

The first visit consisted of measuring the heart rate measures (RHR and HRV) twice. RHR was 79.1 beats per minute (bpm) on the first measurement and then decreased (improved) to $76.1 \mathrm{bpm} 10$ minutes later in the second measurement (Figures 1-3). The latter measurement is approximately the healthy average for the patient's age and gender [13]. HRV was 29.4 milliseconds (ms) on the first measurement and $34.8 \mathrm{~ms}$ on the second (Figures 1-3) This latter HRV number also is the healthy average for females her age [14].

MFD revealed a wide differential of 0.9 degrees Fahrenheit 0.9 degrees warmer on the left side; Figure 4). The normative average for this area is approximately 0.3 degrees F or smaller [15]. Only one temperature measurement was performed on this visit.

Upon inquiry, the patient indicated her position of sleep was prone. Obviously, this means the head is turned, which is not an issue in itself, but for hours at-a-time with the head turned can be an issue. The palpation exam revealed a slight misalignment of the atlas.

Since the heart rate findings (RHR and HRV) for the second neurological measurement during this visit showed improvement compared to the first, I decided my service (of spinal adjustment) was not needed this visit. The patient was simply advised to: a) try to sleep on her side rather than on the stomach, and b) return the next day at the same time (for consistency of measurement purposes) to monitor progress or lack thereof (Figure 1a \& Figure 1b).

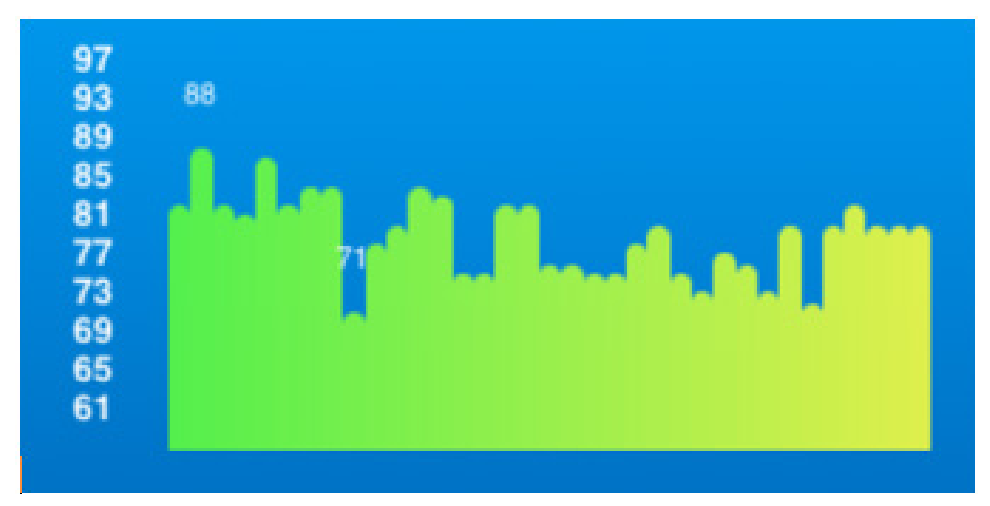

Figure 1a: Visit 1, heart rate reading \#1. RHR = $79.1 \mathrm{bpm}, \mathrm{HRV}=29.4 \mathrm{~ms}$. 


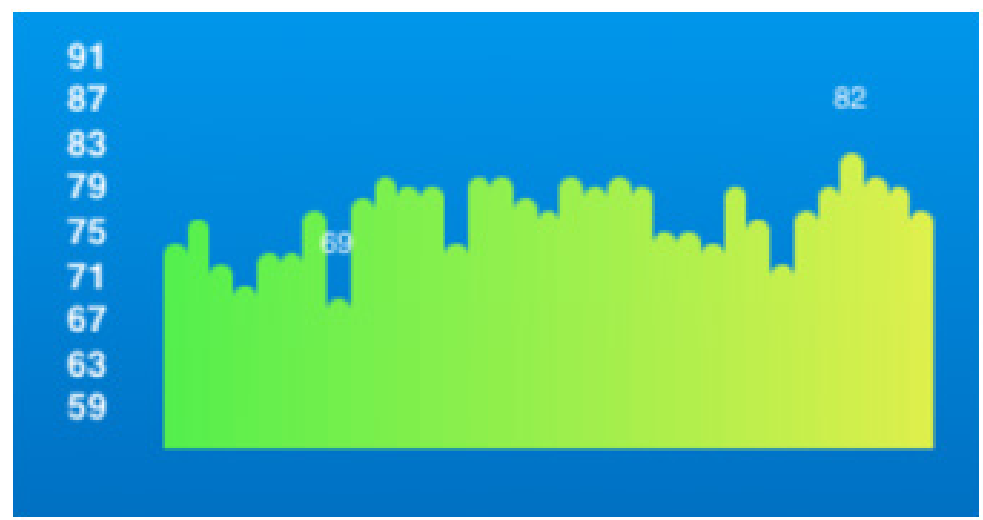

Figure 1b: Visit 1, heart rate reading \#2. RHR $=76.7 \mathrm{bpm}, \mathrm{HRV}=34.8 \mathrm{~ms}$.

\section{Visit 2 of 2}

The patient reported that a few hours following the first visit she felt a "pop" in her neck and immediately experienced substantial symptomatic relief. Additionally, that night she was able to sleep on her side to some extent and noticed further relief the next day.

The atlas continued to show some misalignment this visit. However, the more important finding was the continued ANS improvement, evidenced by further reduction in RHR (to $73.8 \mathrm{bpm}$ ) and further increase (improvement) in HRV (to $45.1 \mathrm{~ms}$, Figures 2,3); and MFD switching warmer sides (from left-to-right, Figure 4). Consequently, intervention (spinal adjustment) was again not indicated and therefore not given. The patient was: a) advised to continue sleeping on her side, and b) encouraged to return for periodic spinal examinations, even if asymptomatic, as neurological disturbance from misalignment can be present without symptoms (Figures 2,3).

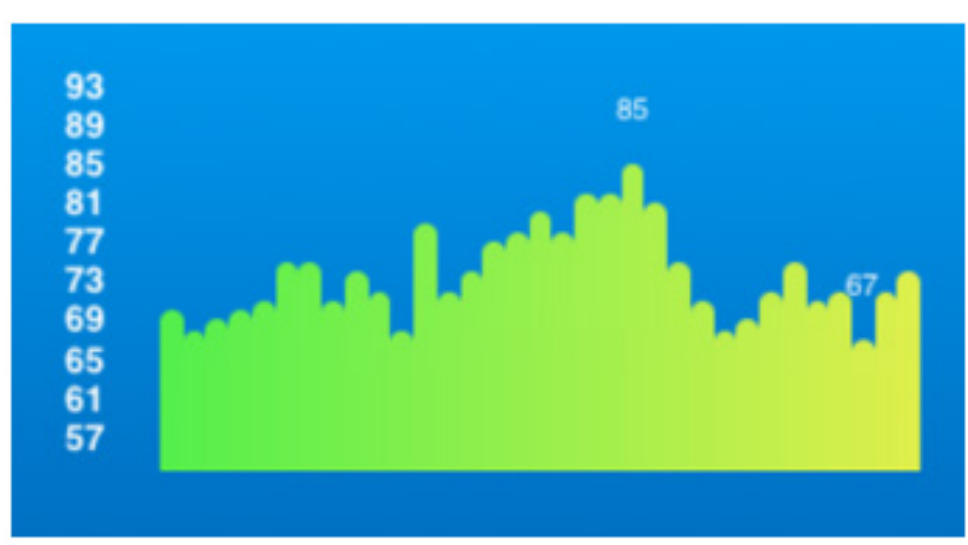

Figure 2: Visit 2, heart rate reading \#3. RHR $=73.8 \mathrm{bpm}, \mathrm{HRV}=45.1 \mathrm{~ms}$.

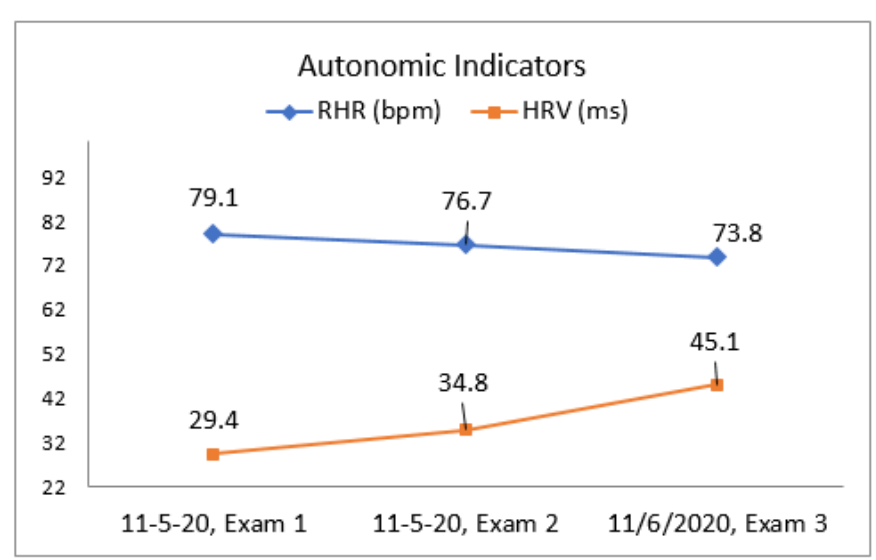

Figure 3: Heart rate values

RHR is resting heart rate. HRV is heart rate variability. Both measures showed improvement from exam 1 to 2 , and from exam 2 to 3 , where RHR decreased and HRV increased. 


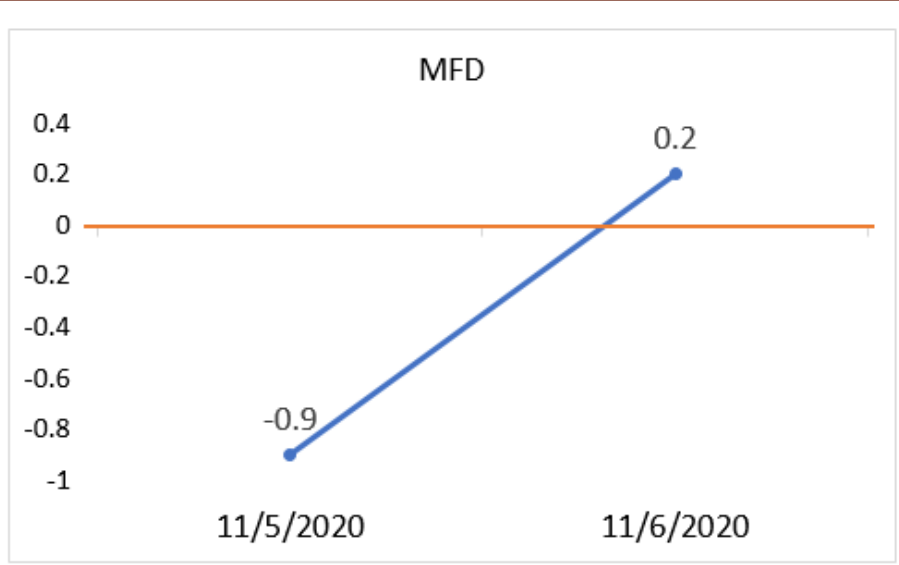

Figure 4: Mastoid fossa temperature differentials (MFD).

Orange line is the zero line. Negative scaled numbers represent warmer readings on the left side, positive numbers represent warmer on the right side.

\section{Follow-up}

In checking on the patient by phone 2 and 4 weeks later she noted continued symptomatic improvement, with only some temporary return of the symptoms if and when stomach sleeping inadvertently occurred. And eight weeks after her office visits, the patient stated she was "doing great."

\section{Discussion}

Regarding the persistent misalignment finding: In the neurologically focused practice, a slight misalignment in the spine does not require spinal adjustment when neurological assessment looks good. Such misalignments, when not accompanied by neurological disturbances, are likely clinically insignificant [16]. Additionally, palpation by hand may not be sensitive enough to detect a possible slight improvement in alignment that may have occurred by the time of this patient's second visit.

Correct timing for an adjustment, given only when neurologically indicated is a key factor for successful chiropractic care in my practice [2]. In this case it appears that the patient was already undergoing an internal, self-adjustment of a cervical spine misalignment, evidenced by improving autonomic indicators, and then culminating perhaps with the "pop" in the neck she felt between the two visits. When the patient is undergoing this kind of self-progress, I prefer to stay out of the way by not providing intervention such as a spinal adjustment. In the future the patient may need an adjustment, but so far, so good. As the not-well-known cliché goes, sometimes the best thing to do is nothing.

\section{Acknowledgement}

I am grateful to the patient for allowing me to tell her story here.

\section{Conflict of Interest}

No conflict of interest.

\section{References}

1. Usman Zulfiqar, Donald A Jurivich, Weihua Gao, Donald H Singer (2010) Relation of high heart rate variability to healthy longevity. The American Journal of Cardiology 105(8): 1181-1185.

2. Hart J (2019) Optimal level of heart rate variability for spinal adjustment: A case report. Journal of Contemporary Chiropractic 2(1): 103-108.

3. Carney RM, Freedland KE, Veith RC, Cryer PE, Skala JA, et al. (1999) Major depression, heart rate, and plasma norepinephrine in patients with coronary heart disease. Biol Psychiatry 45: 458-463.

4. Urbank D, Podgórski M, Mazur G (2018) Heart rate variability-clinical significance. Family Medicine \& Primary Care Review 20(1): 87-90.

5. Campos M (2017) Heart rate variability: A new way to track well-being. Harvard Health Publishing. Harvard Medical School.

6. Gibbon C, Seay C, Hart J (2007) Reliability of a temporal artery thermometer used for mastoid fossa readings. Journal of Chiropractic Medicine 6(2): 66-69.

7. Hsia J, Larson JC, Ockene JK, Sarto GE, Allison MA, et al. (2009) Resting heart rate as a low tech predictor of coronary events in women: prospective cohort study. British Medical Journal 338: 577-580.

8. Zaproudina N, Varmavuo V, Airaksinen O, Narhi M (2008) Reproducibility of infrared thermography measurements in healthy individuals. Physiology Measurement 29: 515-524.

9. Altini $M(2013)$ Heart rate variability Logger - app details.

10. Plews DJ, Scott B, Altini M, Wood M, Kilding AE, et al. (2017) Comparison of heart rate variability recording with smart phone photoplethysmographic Polar H7 chest strap and electrocardiogram methods. Int J Sports Physiol Perform 12(10): 1324-1328.

11. Vescio B, Salsone M, Gambardella A, Quattrone A (2018) Comparison between electrocardiographic and earlobe pulse photoplethysmographic detection for evaluating heart rate variability in healthy subjects in short- and long-term recordings. Sensors (Basel) 18(3): 844.

12. Hart J (2010) Mastoid fossa temperature differential analysis using the Thermofocus instrument and standard deviation analysis: A case report. Journal of Vertebral Subluxation Research 1-4.

13. Ostchega Y, Porter KS, Hughes J, Dillon CF, Nwankwo T (2011) Resting pulse rate reference data for children, adolescents, and adults: United States, 1999-2008. Natl Health Stat Report 24(41): 1-16.

14. Kim GM and Woo JM (2011) Determinants for heart rate variability in a normal Korean population. J Korean Med Sci 26(10): 1293-1298.

15. Uematsu S, Edwin DH, Jankel WR, Kozikowski J, Trattner M (1988) Quantification of thermal asymmetry. Part 1: Normal values and reproducibility. J Neurosurg 69(4): 552-555.

16. Hart JF (2007) Structural problems of the spine do not necessarily require intervention. J Can Chiropr Assoc 51(1): 8-13. 